\title{
Fracture Toughness of Resin Based Composites, Impregnated with Silver Nanoparticles and Bioactive Glass
}

\author{
Amjad Hanif ${ }^{1}$ \\ BDS, MSc \\ Fazal Ghani ${ }^{2}$ \\ BDS, MSc, FDSRCPS, PhD
}

OBJECTIVE: To investigate the effect, on fracture toughness (KIc), of impregnating silver nanoparticles (AgNPs) and bioactive glass (BAG) in resin based composites (RBCs).

METHODOLOGY: During the period from August 2016 to May 2018, this study was performed at Peshawar Dental College (Pakistan) and Montreal University (Canada), using; a commercial RBC and experimental RBCs with or without BAG content (5-15wt\%), and AgNPs (0.009\%). Standardized specimens ( $\mathrm{n}=6)$ were made in each of five RBCs (G1-G5). AgNPs were synthesized and characterized by uv-vis spectroscopy. With universal testing machine, the KIc for RBCs specimens was computed. SEM and dynamic light scattering (DLS) was used to assess the size and form of the prepared silica. One-way ANOVA and Tukey post hoc test were used for data analyses.

RESULTS: KIc values varied both within and between RBCs groups. The commercial RBC had highest mean $K I c(\mathrm{G} 1=1.03+0.24)$. Mean KIc values for the experimental RBCs were; G2 $=0.69 \pm 0.14, \mathrm{G} 3=0.9 \pm 0.13, \mathrm{G} 4=0.9 \pm 0.14$ and G5 $=0.69 \pm 0.13$. The only RBC groups that had statistically significant variations between their mean KIc values were; G1-G2 ( $\mathrm{p}=0.017$ ) \& G1-G5 ( $\mathrm{p}=0.017$ ). SEM and DLS analysis of synthesized silica particles having round shape and sizes of $0.9-1 \mu \mathrm{m}$. Uv-vis spectroscopy of AgNPs showed round shape with size up to 20nm.

CONCLUSION: The KIc of the experimental RBCs with BAG (5-10 wt\%) and AgNPs (0.009\%) was not significantly different than the commercial RBC.

KEYWORDS: Resin based composites, RBCs, Silver nanoparticles, AgNPs, Bioactive glass, BAG, Re-mineralizing resin based composite, Fracture toughness, KIc

HOW TO CITE: Hanif A, Ghani F. Fracture toughness of resin based composites, impregnated with silver nanoparticles and bioactive glass. J Pak Dent Assoc 2020;29(4):179-184.

DOI: https://doi.org/10.25301/JPDA.294.179

Received: 30 June 2020, Accepted: 25 August 2020

\section{INTRODUCTION}

$\mathrm{R}$ esin based composites (RBCs) have multiple applications in dental practice including; direct restorations, inlays, onlays, core build-up, fissure sealing and orthodontic bracket bonding. ${ }^{1}$ With the use of appropriate adhesive technique and their ease of handling, adequate bonding of RBCs to both enamel and dentin can be achieved. ${ }^{2}$ However, an associated drawback with RBC use is that they harbor more microorganisms than some other restorative materials. ${ }^{3}$ A common reason for replacing $\mathrm{RBC}$ restorations has been secondary caries occurring at the restoration-tooth interface. Polymerization shrinkage during setting reaction as well as cyclic mechanical loading may result in the formation of marginal gap and micro-leakage and hence the harboring of microorganisms. ${ }^{4}$ Resin pores

\footnotetext{
1. Assistant Professor, Department of Dental Material, Peshawar Dental College.

2. Professor, Department of Prosthodontics, Peshawar Dental College.

Corresponding author: "Prof. Dr. Fazal Ghani” < fazalg55@ hotmail.com >
}

also allow bacteria inflicted plaque to cause infection. Since the initiation of secondary caries at the tooth-restoration interface frequently occurs in RBCs, therefore it is important to develop innovative RBCs that possess antibacterial properties as well as self-repairing ability through re-mineralization. ${ }^{5}$

The adding of various types of releasing or slow releasing agents has been considered to inculcate antibacterial properties in RBCs. These include silver, iodine, zinc, antibiotics, chlorhexidine and chitosan. ${ }^{6-7}$ For sustained antibacterial properties, immobilized antimicrobial agents have also been added to RBCs. ${ }^{6}$ Among these agents, silver or fillers implanted with silver ions have exhibited a promising antibacterial property without affecting the strength and mechanical characteristics of RBC. Silver containing biomaterials have exhibited effective antimicrobial activity against oral pathogens such as streptococcus mutans, candida albicans, lactobacillus acidophilus. ${ }^{8}$

Re-mineralizing effect can be achieved with the addition of unsalinized BAG filler to RBCs. BAG 45S5 represents 
the composition which is widely employed in orthopedics because of its ability to form hydroxyapatite crystals in physiological environment. The precipitation of hydroxyapatite can also be achieved in a methacrylate based composite material containing bioactive glass. ${ }^{9}$ Several studies have investigated new bioactive and antimicrobial restorative materials for prevention of recurrent decay. However, the majority of these materials exhibit inferior physical properties. ${ }^{6}$ Silanization of filler particles has been shown to improve the mechanical properties but leads to decline in bioactivity. Appropriate inculcation of bioactivity without affecting the mechanical properties of RBCs is an issue yet to be ascertained. ${ }^{9}$

Previous work has reported that some mechanical properties could decline with the addition of calcium phosphate bioactive fillers to the RBCs. ${ }^{10}$ This finding was confirmed by a recent study that investigated some other properties of experimental and commercial RBCs. The experimental RBCs included those with and without a fixed proportion of AgNPs and varying amounts of BAG. ${ }^{11}$ However, this study did not look at the possible effect, on KIc, of the AgNPs and BAG impregnations in the experimental RBCs. KIc is an important mechanical property that refers to the opposition of a brittle material to the proliferation of flaws under an applied stress. It can be related to the fatigue resistance of the material. KIc relies on the chemical composition and physical properties of the components of restorative material. ${ }^{12}$

The present study aims to investigate the effect, on KIc, of adding a fixed amount of AgNPs and various proportions of BAG (5-15\%) in experimental RBCs. The mentioned additions, in the experimental RBCs, will be made to ensure each of the experimental RBCs maintain a $70 \%$ total filler content. We hypothesized that a larger proportion of $\mathrm{BAG}$ substituting the filler content in the RBC would result increased KIc.

\section{METHODOLOGY}

The present study is part of a multiphase research project comprising the synthesis and characterization of AgNPs and BAG and their incorporation to result some experimental RBCs. ${ }^{11,13-15}$ The study protocol and the publication of results were approved by the institute and university research and ethics boards (Riphah/26/17/011 March 03, 2016 and PRIME/ IRB/2019-179). The experimental work was conducted during the period August 2016-May 2018, at the Department of Dental Materials, Peshawar Dental College, (Pakistan) and Chemistry Department, Montreal University in Canada. The pilot phase for the research project / protocol to perfect the experimental resin manufacturing was done in the local setting. Once it was approved, then the further work including the fabrication of the specimens and their testing was done in the overseas setting.

The materials used for the synthesis of experimental RBC and their modifications are given in Tables 1-2. The proprietary RBC was used to make specimens to belong to the control group (G1). The unmodified experimental RBC was used to prepare specimens to comprise the G2 group. The experimental RBCs having $0.009 \%$ AgNPs and 5, 10 and $15 \mathrm{wt} \%$ of BAG-45S5 respectively to substitute the silica particles were used to fabricate specimens to make groups designated as G3, G4 and G5 respectively. First both the resin mixture and filler particles were mixed manually in a plastic container. Then the material was transferred to three roll mill (Exakt, TRM, Norderstedt, Germany) to obtain a homogenous material. In each of the five RBC groups, there were six specimens. Details of the various RBCs, their content are given in Table 2.

A single-edge notched beam (SENB) test was employed to calculate the KIc for the RBC specimens. The notch in the specimen was created by using the custom made threepiece brass mould used for fabricating the specimens. The notch to have a standardized dimension and location in each specimen was facilitated with a sharp blade-like v-shaped

Table. 1: Materials used for the synthesis of experimental RBCs

\begin{tabular}{|c|c|c|}
\hline Materials & Manufacturer & Batch No \\
\hline $\begin{array}{c}\text { Bisphenol Glycidyl } \\
\text { methacrylate (Bis-GMA) }\end{array}$ & Sigma Aldrich USA & 806-321 \\
\hline $\begin{array}{l}\text { Triethylene glycol } \\
\text { di-methacrylate } \\
\text { (TEGDMA) }\end{array}$ & Sigma Aldrich USA & STBG35210V \\
\hline Camphoroquinone (CQ) & Sigma Aldrich USA & A0097555 \\
\hline $\begin{array}{c}\text { Ethyl-4-(di-methylamino) } \\
\text { benzoate (EDMAB) }\end{array}$ & Alfa Aesar USA & МKCB6154 \\
\hline Silver 2 ethyl hexanoate & $\begin{array}{c}\text { Strem Chemicals } \\
\text { USA }\end{array}$ & A1918077 \\
\hline Bioactive Glass (45S5) & $\begin{array}{c}\text { Denfotex Research } \\
\text { Ltd UK }\end{array}$ & 1608108 \\
\hline $\begin{array}{l}\text { 2-tert butyl amino ethyl } \\
\text { methacrylate (TBAEMA) }\end{array}$ & SigmaAldrich, USA & MKCB2542V \\
\hline $\begin{array}{c}\text { Tetra ethyl orthosilicate } \\
\text { (TEOS) }\end{array}$ & Sigma Aldrich, USA & MKBP8202V \\
\hline $\begin{array}{c}\text { Ammonium Hydroxide } \\
\left(\mathrm{NH}_{4} \mathrm{OH}_{3}\right.\end{array}$ & Sigma Aldrich, USA & 45304 \\
\hline Nano silica (50nm) & Aerosol OX & 154052345 \\
\hline
\end{tabular}


ridge in the mould surface. There was no direct involvement of the operator in the production of the notch in the specimens.

The specimens fabrication as well as the testing procedure were that of the American Standards for Testing Materials (ASTM).${ }^{16}$ Rectangular bar specimens $(n=6)$ in each $\mathrm{RBC}$ having pre-specified dimensions (height $=4 \mathrm{~mm}$, width $=2$ $\mathrm{mm}$ and length $=25 \mathrm{~mm}$ (Figure 1 ) were fabricated using a three-piece brass split mold so that on completion of curing,

Table. 2: RBC groups, their Klc values and statistical analyses

\begin{tabular}{|c|c|c|}
\hline Group & RBC Details & $\begin{array}{l}\text { Mean } K l c \text { Values } \pm \\
\text { S. Dev }\end{array}$ \\
\hline G1 & $\begin{array}{l}\text { Commercial RBC(3M Filtek } \\
\text { Z250XT) }\end{array}$ & $1.03 \pm 0.24$ \\
\hline G2 & $\begin{array}{l}\text { Experimental RBC ( } 70 \% \text { wt } \\
\text { filler, } 0 \% \text { BAG, } 0 \% \text { AgNPs) }\end{array}$ & $0.69 \pm 0.14$ \\
\hline G3 & $\begin{array}{l}\text { Experimental RBC (70\% wt } \\
\text { filler,5\%BAG,0.009\%AgNPs) }\end{array}$ & $0.90 \pm 0.13$ \\
\hline G4 & $\begin{array}{l}\text { Experimental RBC (70\% wt } \\
\text { filler, } 10 \% \text { BAG,0.009\%AgNPs) }\end{array}$ & $0.90 \pm 0.14$ \\
\hline G5 & $\begin{array}{l}\text { Experimental RBC(70\% wt } \\
\text { filler,15\%BAG, } 0.009 \% \mathrm{AgNPs})\end{array}$ & $0.69 \pm 0.13$ \\
\hline \multicolumn{2}{|c|}{$\begin{array}{l}\text { Groups with significant variations } \\
\text { between mean values. }\end{array}$} & $\begin{array}{l}\text { G1-G2 }(p-v a l u e= \\
0.017) \& \text { G1-G5 } \\
(p=0.017) .\end{array}$ \\
\hline \multicolumn{2}{|c|}{$\begin{array}{l}\text { Groups with insignificant variations } \\
\text { between mean values. }\end{array}$} & $\begin{array}{l}\text { G1-G3, G1-G4, G2- } \\
\text { G3, G2-G4, G2-G5, } \\
\text { G3-G4 G3-G5, G4- } \\
\text { G5 }\end{array}$ \\
\hline
\end{tabular}

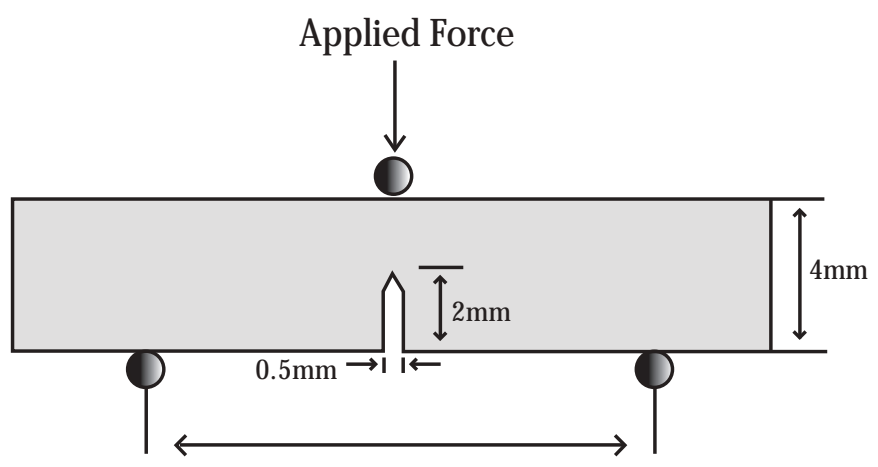

Figure. 1: Diagrammatic representation of the dimensions of the specimen and illustration of the fracture toughness test. ${ }^{15}$

the bar could be removed from the mold without the application of force. The mold was filled manually with the help of stainless steel spatula. The material was transferred into the mold in 1-2 mm increments. Each addition was polymerized for 20 -sec with light-curing machine with an output of $750 \mathrm{~mW} / \mathrm{cm}^{2}$ and a wavelength of about $470 \mathrm{~nm}$. On curing, each specimen was separated from the mold and further cured on each side for another 10-sec. Excess material on the sides of the specimen was then removed with 1000grit sandpaper. Before calculation of the KIc, specimens considered suitable and defect-free remained stored in distilled water at $37 \mathrm{C}$ for a minimum of 24 hours. Three-point bending configuration was used to determine KIc in ambient temperature using UTM (Instron.5565 USA) at a cross head speed of $0.75 \mathrm{~mm} / \mathrm{min}$ and pre-set load of $50 \mathrm{~N}$.KIC was determined with the help of equation. ${ }^{16}$

$\mathrm{K}_{\mathrm{IC}}=\left(\frac{P L}{b w 1.5}\right) f\left(\frac{a}{w}\right)$

Where

$$
f\left\{\frac{a}{w}\right\}=\frac{3}{\alpha}\left(\frac{a}{w}\right)^{0.5}\left\{1.99-\left(\frac{a}{w}\right)\left(1-\frac{a}{w}\right) \times\left[2.15-3.93 \frac{a}{w}\right]\right.
$$

$\left.\left.\left.2.7\left(\frac{a}{w}\right)\right\}^{2}\right]\right\}$

Where $\alpha=2(1+2 \mathrm{a} / \mathrm{w})(1-\mathrm{a} / \mathrm{w}) 2 / 3, \mathrm{KIC}=$ Stress Intensity Factor, $\mathrm{L}=$ distance between supports, $\mathrm{w}=$ width of the specimen, $\mathrm{b}=$ thickness of the specimen and $\mathrm{a}=$ crack length.

The shape and size of silica particles and BAG was determined by using DLS (Malvern Zetasizer UK). AgNPs were synthesized following the standard procedure ${ }^{13}$ and were characterized with the help of uv-vis spectroscopy (Cary uv-vis Agilent technologies, USA). SEM analysis (JEOL, Model JSM-7600F, Japan) of fractured surface through the bulk of samples following mechanical characterization was used to observe the filler distribution and morphology.

Statistical package for social sciences SPSS software version 19 for windows was used for data analysis. Mean and standard deviation for KIc values for specimens in all the five groups were computed. Data analyses, using ANOVA and Tukey's post hoc test was made to compare the level of significance of the differences from the mean values of KIc among the RBC groups. For the difference to be statistically significant, the $\mathrm{P}$ value was set as $<0.05$.

\section{RESULTS}

The mean $K I c$ values for all the $\mathrm{RBC}$ groups are given in Table 2. It can be seen that the commercial $\mathrm{RBC}(\mathrm{G} 1)$ exhibited higher value. It can also be seen that specimens in the experimental RBC with no BAG and AgNPs (G2) or those containing AGNPs $(0.009 \%)$ and BAG content of $15 \%$ had lower mean KIc values. The mean KIc values for both the experimental RBCs (G3 and G4) can be ranked next to the mean KIc value exhibited by the commercial RBC (G1). The significance or otherwise of the differences between the mean KIc values between the various RBC groups are also 
given in Table 2 . These show that the only mean KIc values that were statistically significantly different were those between the RBC groups; G1-G2 and G1-G5.

The SEM images of the representative surfaces of the fractured RBC specimens are shown in Figure 2 (a-c). The silica particles were of round shape (Figure 2a) and their sizes ranged from 0.9 to $1.0 \mu \mathrm{m}$. Average size of BAG particles determined by DLS was $512 \mathrm{~nm}$. Figure $2 \mathrm{~b}$ shows that some of the filler particles appear detached from the matrix, leaving empty spaces indicating weak bonding between the filler and the matrix. The SEM image

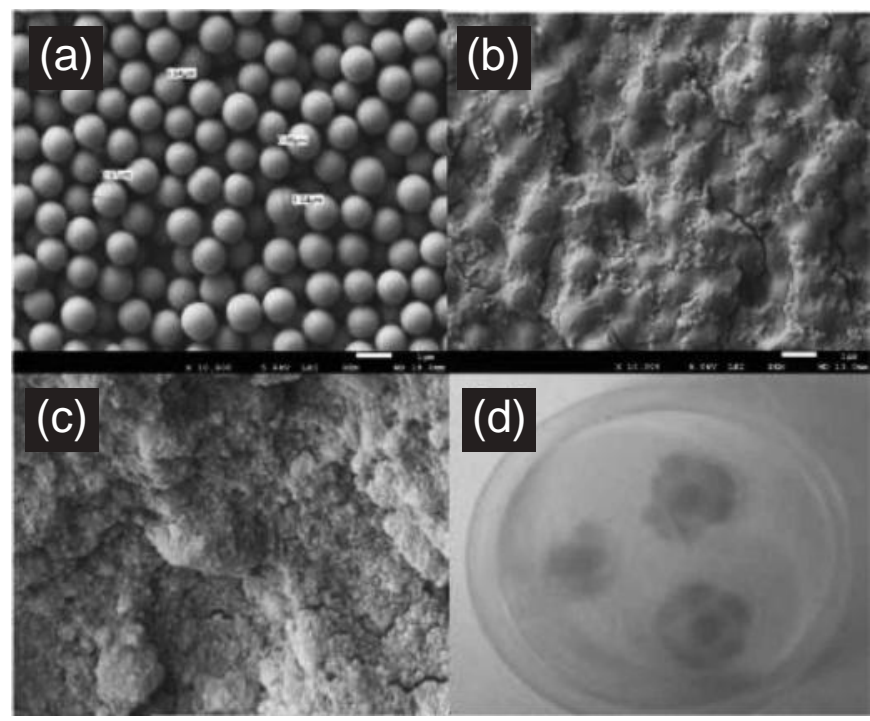

Figure. 2: (a) SEM of synthesized silica particles at voltage of $5 \mathrm{kv}$ and 10,000 magnification. (b) SEM of experimental resin composite (G3) showing surface morphology. (c) SEM of commercially available resin composite (G1) showing surface morphology. (d) Thin films of photo-polymerized resin containing AgNPs.

(Figure 2c) of a specimen belonging to G1 group shows that the filler size is much smaller than the particles size in the specimens belonging to the other RBC groups. Surface appears rougher and filler particles are more densely packed when compared to the other RBC groups.

\section{DISCUSSION}

The Klc values (Table 2) for specimens in the various RBC groups were calculated by using a single-edge-notchedbeam (SENB) test. ${ }^{16}$ This test has been considered reliable and valid in addition to being simple in terms of making specimens. ${ }^{17}$ Three-or four-point bending equipment is used for measuring the fracture toughness using a single-edge notched-beam. Both the methods are being commonly used in dental materials research. ${ }^{18}$ As mentioned earlier, the notch was created in a standardized manner in each specimen with no direct involvement of the operator in its production.

To provide valid and realistic comparison of the data, this study also included specimens made in commercial restorative material (Filtek $3 \mathrm{M}, \mathrm{Z} 250^{\mathrm{xt}}$ ) so that the KIc value could act and provide as control group. ${ }^{19}$ The specimens made in the commercial RBC(G1) exhibited higher mean $K I c$ value. The commercial RBC contained $82 \%$ filler in comparison to the $70 \%$ total filler content in the RBC specimens in the rest of the groups. A variety of filler percentages to resin matrix have been added with the range of $70-75 \%$. With the $70 \mathrm{wt} \%$ filler content, the viscosity of the resin paste was adequate as well as its handling. Most of the studies are in agreement that $K l c$ toughness increases with an increase in the amount of filler. Ilea et $\mathrm{al}^{18}$ reported that the KIc was highest in the RBCs containing higher percentage of filler and not dependent on the extent of polymeric conversion in the resin matrix. The filler phase present in RBCs distributes the applied force into many components leading to deviation of the crack path and thus hindering the proliferation of crack. ${ }^{17}$ Figure $2 \mathrm{c}$ belonging to the RBC specimens from the G1 shows that the particle size of filler is smaller than the experimental RBC and the particles are well-condensed without any signs of detachment of filler from the matrix indicating excellent silanization of filler particles. Some studies have reported that matrix/particle detachment can be harmful to the fatigue and fracture performance of RBCs. Figure $2 b$ shows small depressions indicating sites of detachment of filler particles from the matrix which also might be responsible for low KIc values of the experimental RBCs when compared to those made in the proprietary $\mathrm{RBC}$.

The data in Table 2, show relatively higher KIc values for both the G3 and G4 RBC specimens compared to those in the $\mathrm{G} 2$ group. This indicates that BAG addition imparted a beneficial effect. The data also show that further increase of the BAG proportion in the experimental $\mathrm{RBC}$ and the specimens made in it (G5) resulted in a decrease in Klc though to a statistically insignificant extent. A likely explanation for this finding could be the existence of increased BAG aggregates in the resin matrix without chemical adhesion between them. Smaller BAG content in the experimental RBCs, could better deflect crack from the front originating from the notch in specimen. In fact, it has been shown that the stress intensity factor $(\mathrm{Klc})$ will decrease at the tip of crack with the change of direction, halting its unstable growth. ${ }^{20}$ Moreover, there might be trans-particle fracture due to which more energy would be needed to break the BAG agglomerate leading to increase in fracture toughness. ${ }^{20}$ However, this phenomenon might not function at higher concentration of the BAG in the resin matrix. This was confirmed from the findings of the present study where an 
increased $\mathrm{BAG}$ content in the $\mathrm{RBC}$ used for making specimens in the group G5 did not result in further increase in $K l c$ but rather decreased it. While the exact cause for this has yet to be identified, but it could likely be due to increased amount of flaws, voids or porosities. ${ }^{18}$ Chatzistavrou, et $\mathrm{al}^{6}$ have also reported insignificant difference in the mechanical properties of specimens compared to those in the control samples made in the RBC having silver doped BAG with BAG content of 5, $10 \& 15 \%$ respectively. However, in that study mechanical properties were characterized by the method of micro-tensile test that measured total bond strength of dentin.

Uv-vis spectroscopy of photo polymerized resin films indicated nano size (less than 20nm) and round shape of silver particles. Based on a previous study ${ }^{13}$ that reported antibacterial property of AgNPs, $0.009 \%$ AgNPs $(0.03 \%$ silver in resin) was incorporated in RBC. AgNPs with high surface area and small size have adequate antibacterial activity at low filler level. Antibacterial properties of AgNPs at such low level $(0.009 \%)$ in RBC need to be investigated in future studies. Low silver salt concentrations $(\leq 0.028 \%)$ results in minimal clumping. Since the concentration used in this study is extremely low $(0.009 \%)$ which is lower than $0.028 \%$, it can be predicted that such a concentration of AgNPs wouldn't have adversely affected the mechanical properties of RBC. However, it has been found that in-situ reduction of silver ion competes with free radicals produced by light activation. This might result in increased inactivation of proliferating chain and thus leading to decreased mechanical properties. ${ }^{13}$

In this study mode I fracture toughness of the RBC specimens was determined which is particularly important because it is the lowest stress at which crack proliferation can initiate. Clinically this opening (tensile) mode of failure is most closely linked to restorative materials in terms of fracture type. Klc values may vary depending upon the mode of fracture toughness and test method used. ${ }^{21}$ The results of the single beam notch test are very sensitive to notch depth and width and hence making comparison with other studies difficult. ${ }^{18}$ The $K l c$ values found in this study are within the range (Mode 1, Fracture toughness, $0.55-1.36 \mathrm{MNm}^{-}{ }^{3 / 2}$ ) reported in another study for commercial RBCs using Brazillian disc method. ${ }^{21}$ The values of fracture toughness calculated by Brazillian disc method are lower than the values determined by single edge $\mathrm{V}$ notch beam test. ${ }^{21}$ Therefore, the fracture toughness values obtained by different methods cannot be compared with each other.

Notwithstanding the individual variation and the apparently lower fracture toughness values for the various experimental $\mathrm{RBC}$ containing different proportions of $\mathrm{BAG}$ and the fixed amount of AgNPs in comparison to the control
RBC, keeping in view the beneficial antibacterial and remineralizing effect of the AgNPs and BAG in the experimental RBC, they could be further explored for use in restoring non-stress bearing location (class 3 and 5 cavities). For stress bearing areas such as class 2 and 4 cavities, ways for improving fracture toughness of the experimental RBC require further experimentation.

\section{LIMITATIONS}

Among the several limitations of this study, one was the use of brass mold for fabricating RBC specimens. Brass obstructs the penetration of light delivered by the visible light curing unit affecting the polymerization process and the degree of conversion. We minimized this effect by following the protocol of incremental addition and curing of each of the RBC layer placed in the mold. Another point of interest related to this study is that the total filler content used in the preparation of various experimental RBCs was $70 \mathrm{wt} \%$ compared to $82 \%$ in the commercially available RBC (G1). As stated earlier, filler mass fraction has considerable influence on $K l c$, therefore comparison between G1 and the rest of the groups become difficult. Another limitation of this study was that the influence of AgNPs and BAG individually on the Klc values of the RBCs was not determined, making the comparison of the values with other studies difficult which have used either AgNPs or BAG alone as additives in their RBCs.

\section{CONCLUSIONS}

In comparison to control RBC, lower Klc values were reported for the experimental RBCs. However, these values were only significantly lower for the experimental RBCs groups including; G2 (without AgNPs and BAG) or G5 (AgNPs- $0.009 \%$ and BAG $15 \mathrm{wt} \%$ ).

The experimental RBCs containing AgNPs (0.009\%) and BAG (5-10wt $\%)$, because of their established antibacterial and re-mineralizing effect may prove useful for restoring non-stress bearing location in permanent teeth and all cavities including the stress-bearing location in case of deciduous teeth that receive lower masticatory loads.

\section{ACKNOWLEDGEMENT}

Denfotex Research Ltd, London SE1 1UL, UK kindly donated Bioactive Glass for this research.

\section{CONFLICT OF INTEREST}

None declared. 


\section{AUTHORS CONTRIBUTION}

AH: Conceived the idea, designed and conducted the research and prepared the research protocol and contributed to all drafts of the manuscript.

FG: Refined, perfected the experimental protocol, supervised and interpreted the data, revised and edited all the drafts including the final submitted manuscript and accepted responsibility as the corresponding author.

\section{REFERENCES}

1. Cheng L, Weir MD, Xu HHK, Antonucci JM, Lin NJ, Lin-Gibson $\mathrm{S}, \mathrm{Xu} \mathrm{SM}$, Zhou X. Effect of amorphous calcium phosphate and silver nanocomposites on dental plaque microcosm biofilms. J Biomed Mater Res Part B 2012:100B:1378-1386. https://doi.org/10.1002/jbm.b.32709

2. Korkut E, Torlak E, Altunsoy M. Antimicrobial and mechanical properties of dental resin composite containing bioactive glass. J Appl Biomater Funct2016;14(3):e296-301. https://doi.org/10.5301/jabfm.5000271

3. Zhang N, Melo M, Weir M, Reynolds M, Bai Y, Xu H. Do dental resin composites accumulate more oral biofilms and plaque than amalgam and glass-ionomer. Dent Mater 2016;9,888:1-13. https://doi.org/10.3390/ma9110888

4. Khvostenko D, Mitchell JC, Hilton TJ, Ferracane JL, Kruzic JJ. Mechanical performance of novel bioactive glass containing dental restorative composites. Dent Mater. 2013;29:1139-1148.

https://doi.org/10.1016/j.dental.2013.08.207

5. Villegas NA, Compagnucci MJS, Aja MS, Rocca DM, Becerra MC, Molina GF, Palma SD. Novel antibacterial resin-based filling material containing nanoparticles for the potential one-step treatment of caries. J Health Eng 2019;1-8.

https://doi.org/10.1155/2019/6367919

6. Chatzistavrou X, Lefkelidou A, Papdopoulou L, Pavlidou E, Paraskevopoulos KM, Fenno JC, Flanngen S. Bactericidal and bioactive dental composites, Front Physiol 2018;9(Article 103):1-11. https://doi.org/10.3389/fphys.2018.00103

7. Ali S, Sangi K, Kumar N. Exploring Antibacterial Activity and Hydrolytic Stability of Resin Dental Composite Restorative Materials Containing Chitosan. Technol Health Care 2017;25:11-18.

https://doi.org/10.3233/THC-161238

8. Dias H, Bernardi M, Marangoni V, de Abreu Bernardi A, de Souza Rastelli A, Hernandes A. Synthesis, characterization and application of Ag doped $\mathrm{ZnO}$ nanoparticles in a composite resin. Mater Sci Eng2019; 96:391-401.

https://doi.org/10.1016/j.msec.2018.10.063

9. Par M, Tarle Z, Hickel R, Ilie N. Polymerization kinetics of experimental bioactive composites containing bioactive glass. J Dent.
2018;76:83-88.

https://doi.org/10.1016/j.jdent.2018.06.012

10. Par M, Tarle Z, Hickel R, Ilie N. Mechanical properties of experimental composites containing bioactive glass after artificial aging in water and ethanol. Clin Oral Investig 2018;23:2733-2741. https://doi.org/10.1007/s00784-018-2713-6

11. Hanif A, Ghani F. Mechanical properties of an experimental resin based composite containing silver nanoparticles and bioactive glass. Pak J Med Sci. 2020;36:776-781.

https://doi.org/10.12669/pjms.36.4.1913

12. Lassila L, Keulemans F, Säilynoja E, Vallittu P, Garoushi S. Mechanical properties and fracture behavior of flowable fiber reinforced composite restorations. Dent Mater2018;34:598-606.

https://doi.org/10.1007/s00784-018-2713-6

13. Cheng YJ, Zeiger DN, Howarter JA, Zhang X, Lin NJ, Antonucci JM, Lin-Gibson S. In situ formation of silver nanoparticles in photo cross-linking polymers. J Biomed Mater Res 2011; 124-131. https://doi.org/10.1002/jbm.b.31793

14. Wang R, Habib E, Zhu X. Synthesis of wrinkled mesoporous silica and its reinforcing effect for dental resin composites. Dent Mater 2017;33:1139-1148.

https://doi.org/10.1016/j.dental.2017.07.012

15. Karabela MM, Sideridou ID. Synthesis and study of properties of dental resin composites with different nano silica particle size. Dent Mater 2011;27:825-835

https://doi.org/10.1016/j.dental.2011.04.008

16. Gan X, Wu T, Zhu Z, Wu X, Liao Y, Yu H, Wang H. Fracture toughness comparison of five indirect resin composites under the effect of thermal cycling, Particul Sci Technol2015;34:194-200. https://doi.org/10.1080/02726351.2015.1058873

17. Al-Haddad A, Roudsari VR, Satterthwaite J. Fracture toughness of heat cured denture base acrylic resin modified with chlorhexidine and fluconazole as bioactive compounds. J Dent 2014;42:180-184. https://doi.org/10.1016/j.jdent.2013.11.007

18. Ilie N, Hickel R, Valceanu A, Huth K. Fracture toughness of dental restorative materials. Clin Oral Investig 2012;16:489-498. https://doi.org/10.1007/s00784-011-0525-z

19. Peskersoy C, Culha O. Comparative evaluation of mechanical properties of dental nanomaterials. J Nanomater 2017;7:1-8. https://doi.org/10.1155/2017/6171578

20. Chiari M, Rodrigues M, Xavier T, de Souza E, Arana-Chavez V, Braga R. Mechanical properties and ion release from bioactive restorative composites containing glass fillers and calcium phosphate nano-structured particles. Dent Mater 2015;31:726-733. https://doi.org/10.1016/j.dental.2015.03.015

21. Watanabe H, Khera S, Vargas M, Qian F. Fracture toughness comparison of six resin composites. Dent Mater 2008;24:418-425. https://doi.org/10.1016/j.dental.2007.06.018 